\title{
The appropriate position of radiology in COVID-19 diagnosis and treatment-current status and opinion from China
}

\author{
Huadan Xue ${ }^{1} \cdot$ Zhengyu Jin $^{1}$ \\ Published online: 26 March 2020 \\ (c) Springer Nature Singapore Pte Ltd. 2020
}

With the epidemic of 2019 novel coronavirus, COVID-19 has spread rapidly from Wuhan throughout the country during the past few months. In recent days, it is reported to be spreading in many other countries including Korea, Japan, Italy, etc. According to WHO's report [1], there's already 79,331 confirmed cases globally, in which 77,262 are from China) until 25th Feb 2020. NCP is a highly contagious disease characterised by a long incubation period and its onset of concealment. This disease has already called attention worldwide. And Chinese government reacted quickly with six versions publication of programme for the diagnosis and treatment of the disease.

As everyone might have noticed, CT was recommended as the major evidence of clinical diagnosis in HuBei province by the National Health and Health Commission of China 5th edition in Feb 5th 2020 [2] but had been eliminated by the next edition on Feb 19th 2020 [3]. One might be interested in the background of this action.

As we all know, RT-PCR assay is the gold standard of COVID-19 diagnosis, however its high false negative detection rate $(30-50 \%)$ due to sampling position and possible low virus load hinders its validation during a special period of time [4]. Several previous reports had already reviewed RT-PCR negative patient cohorts with CT positive results [5, 6].While, Initial CT, as reported, yielded much higher detection rate (98\%) of COVID-19 than first RT-PCR (71\%) $(P<0.001)$ [7].

Also, the RT-PCR operation needs much longer time (2-24 h) than a CT scan (about $5 \mathrm{~s}$ ), not to mention its complicated confirmation process (throat swab first and then lower respiratory sampling if negative, normally twice at least with time interval no shorter than $24 \mathrm{~h}$, hospital to

Zhengyu Jin

jin_zhengyu@163.com

1 Department of Radiology, Peking, Union Medical College Hospital, Shuaifuyuan 1\#, Beijing 100730,

Dongcheng District, China
$\mathrm{CDC}$ and then backwards). For medical workers who are manipulating these procedures, CT scan is also far safer than a throat swab sampling or even lower respiratory tract sampling (noted the latter is recommended in the newest version of Chinese government official notice) [3].

Normally, repeated PCR can reach a certain accuracy rate (reference) if repeated samples were tested. But checking the situation in HuBei province between release of the 4th edition (28th Jan) and the 5th edition (5th Feb) at that time, there were far more patients waiting to be diagnosed and isolated in HuBei province than clinical available RT-PCR test kits. With the peak in the new diagnosed patient curve (Figs. 1, 2), we understood the long patient waiting list outside the hospital needed to be sorted and to be treated at that time in HuBei province. Those with typical CT findings among them, under the guidance of the 5th edition, received timely medical care. This also helped to maximally reduce the risk of community cross infection. After 2 weeks, at time of release of the 6th edition, those big bunch of patients with suspicious COVID-19 infection were successfully collected into hospital. Thus, in the 6th edition, definition of clinical diagnosis was cancelled. Indeed, RT-PCR test is still the most important and is the gold-standard diagnosis for this disease country wide.

However, CT is still one of the important tools in the diagnosis and treatment pathway of COVID-19. Thin slice chest $\mathrm{CT}$ is easy to perform, fast, and is capable of providing valuable information for further diagnosis, while aiding prevention and control of COVID-19.

First, in patients with COVID-19 pneumonia, the most common initial lung findings on chest $\mathrm{CT}$ were small subpleural ground glass opacities (GGO) with which patients could have no symptoms at all including fever. Due to its high sensitivity of COVID-19 diagnosis, CT is the front line of disease detection at the early clinical stage or even pre-clinical stage. Second, CT findings correlate with the severity of COVID-19 and it is also an important tool to perform fast and accurate follow-up evaluation of the treatment 
The curve of daily newly diagnosed COVID-19 case in and outside Hubei province

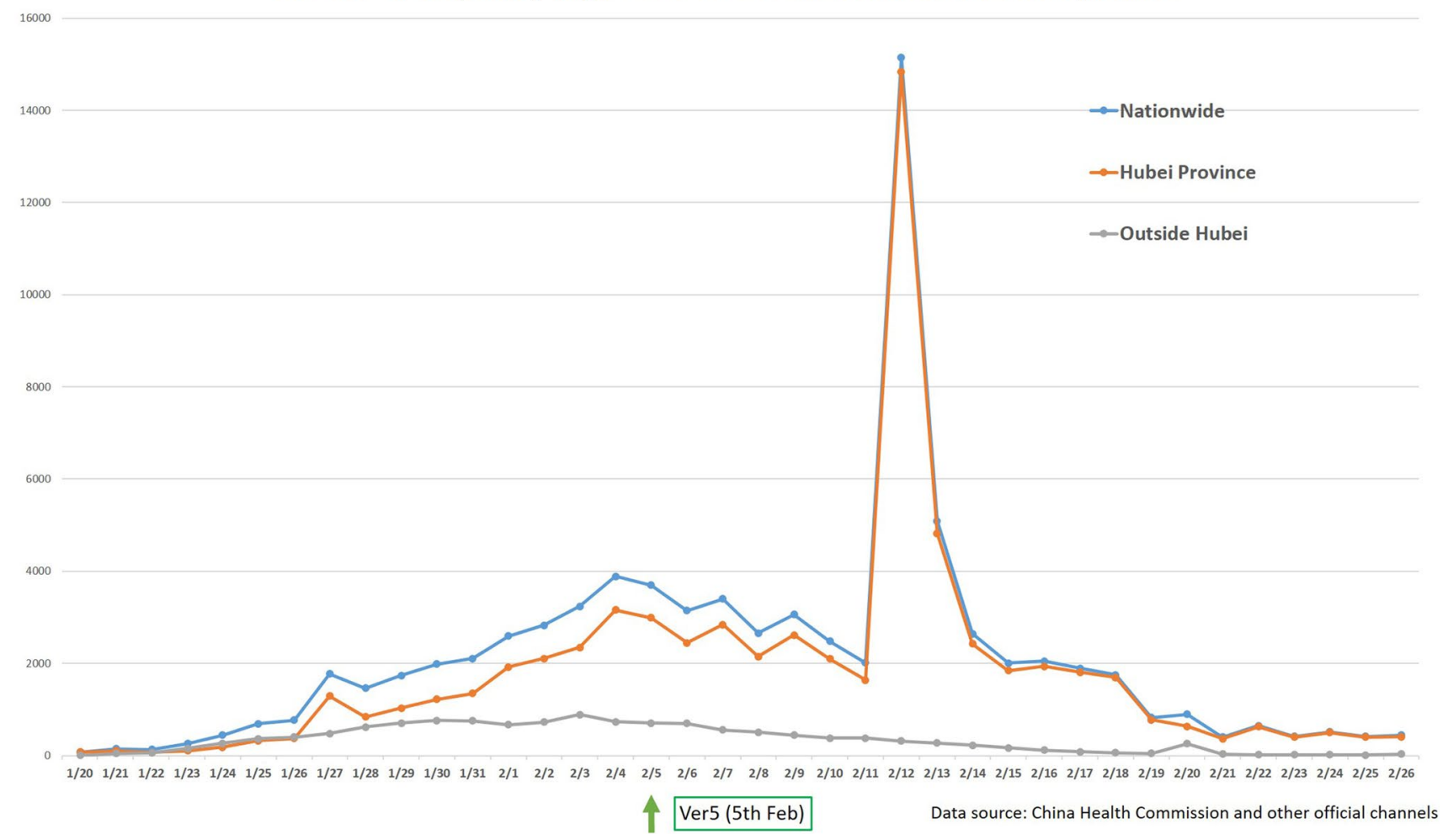

Fig. 1 The curve of daily newly diagnosed COVID-19 case in and outside Hubei province. Following the release of guideline version 5 on 5th February, the main change happened in Wuhan, which is the

outcome. Feng P developed a CT scoring system to quantitatively estimate the pulmonary involvements and it was related with different disease stages especially between the early stage and the progressive stage [8]. Third, CT is one of the important check points for the definition of disease curation. According to the programme for the diagnosis and treatment of novel coronavirus (2019-nCoV) infected pneumonia (the 6th edition) [3], improved CT findings should be checked before the patient is to be discharged from the hospital.

Intended to share their limited but the most updated knowledge about NCP, a group of young radiologists from the Chinese Society of Radiology Youth Committee have developed an article entitled "Progress and Prospect on Imaging Diagnosis of COVID-19" in the past few weeks besides their heavy workload in the prevention and clinical main influencing factor of national cases. The release of ver5 guidelines has little impact on the number of new cases in areas other than Wuhan

treatment for this disease. This review focuses on the aetiology, epidemiology, and clinical symptoms of COVID-19, while highlighting the $\mathrm{CT}$ features of different stages and its differential diagnosis of other lung diseases.

Yet, a number of imaging related questions remains to be answered, including different imaging features in different populations and the relationship between image, pathology and prognosis. What's more, AI might help to improve its imaging quantitative analysis which is also worth further investigation. Overall, this will become a long term hot topic as long as the disease exists.

Hopefully with this, we could provide a comprehensive introduction and analysis of NCP CT imaging so that to help the prevention and treatment of the current outbreak worldwide. 


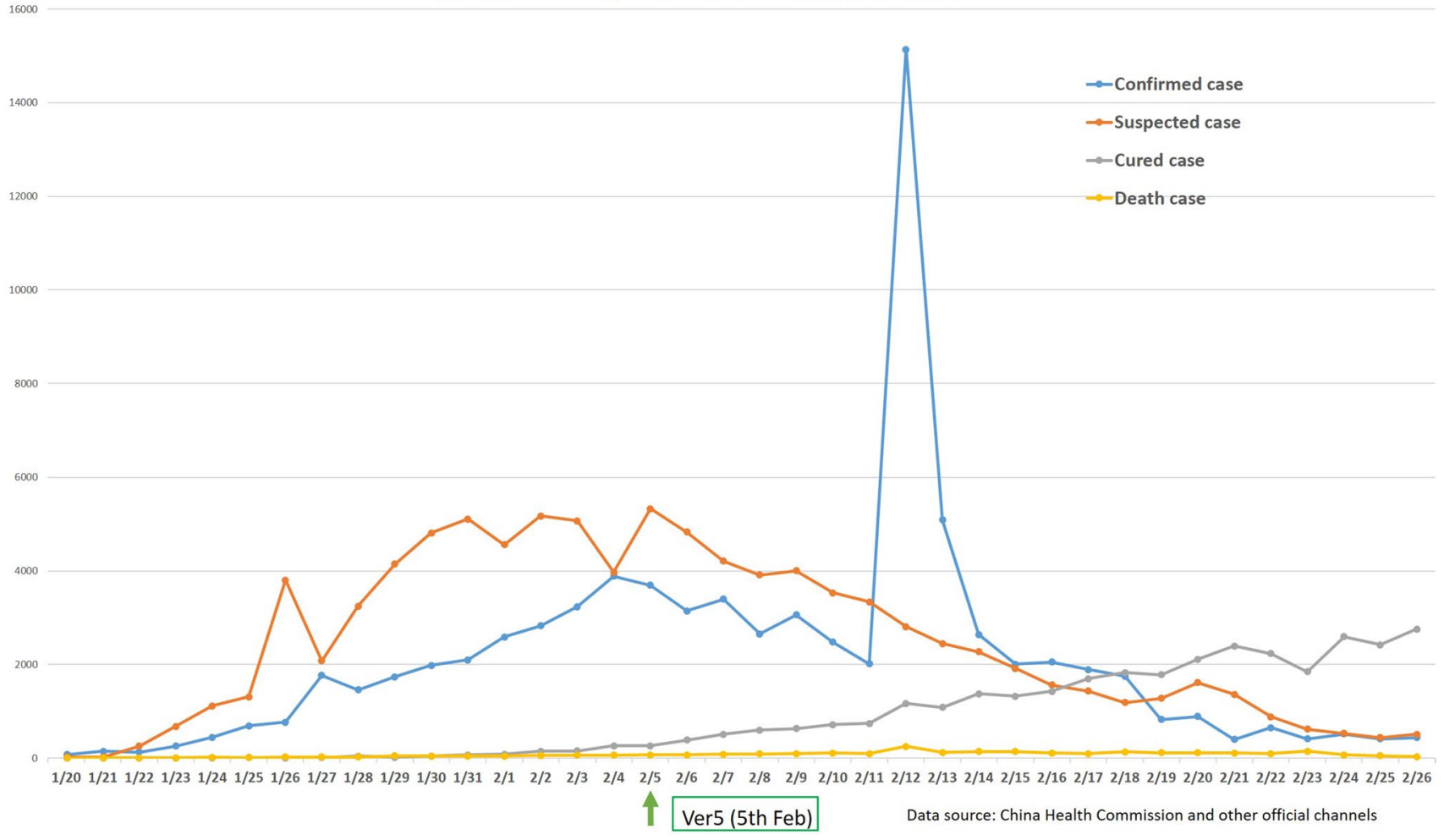

Fig. 2 The curve of daily COVID-19 case changes in China. Note that several days following the release of guideline version 5 in 5 th February there are several changes, firstly, a steep peak of confirmed case showed up in 12th February due to the use of clinical diagnos-

\section{References}

1. Coronavirus disease 2019 (COVID-19) Situation Report - 35 . 2020. https://www.who.int/docs/default-source/coronaviruse/ situation-reports/20200224-sitrep-35-covid-19.pdf?sfvrsn=1ac42 18d_2.

2. General Office of National Health Committee. Office of state administration of traditional Chinese medicine. Notice on the issuance of a programme for the diagnosis and treatment of novel coronavirus (2019-nCoV) infected pneumonia (trial fifth edition). 2020. https://bgs.satcm.gov.cn/zhengcewenjian/2020-02-06/12847 .html

3. General Office of National Health Committee. Office of state administration of traditional Chinese medicine. Notice on the issuance of a programme for the diagnosis and treatment of novel coronavirus (2019-nCoV) infected pneumonia (trial sixth edition). 2020. https://www.gov.cn/zhengce/2020-02/19/content_54809 58.html tic criteria, secondly, the number of suspected cases continued to decline, thirdly, the daily cured cases continued to increase. We can infer that after version 5 , the number of patients on the waiting list to be sorted dropped accordingly

4. World Health Organization. Guidance on regulations for the transport of infectious substances 2019-2020. 2020. https://www.gov. cn/zhengce/2020-02/19/content_5480958.html

5. Xie X, Zhong Z, Zhao W, Zheng C, Wang F, Liu J. Chest CT for typical 2019-nCoV pneumonia: relationship to negative RT-PCR testing. Radiology. 2020. https://doi.org/10.1148/radiol.20202 00343.

6. Huang P, Liu T, Huang L, Liu H, Lei M, Xu W, et al. Use of chest CT in combination with negative RT-PCR assay for the 2019 novel coronavirus but high clinical suspicion. Radiology. 2020. https://doi.org/10.1148/radiol.2020200330.

7. Fang Y, Zhang H, Xie J, Lin M, Ying L, Pang P, et al. Sensitivity of chest CT for COVID-19: comparison to RT-PCR. Radiology. 2020. https://doi.org/10.1148/radiol.2020200432.

8. Pan F, Ye T, Sun P, Gui S, Liang B, Li L, et al. Time course of lung changes on chest CT during recovery from 2019 novel coronavirus (COVID-19) Pneumonia. Radiology. 2020. https:// doi.org/10.1148/radiol.2020200370. 